\title{
Synergistic inhibition of the growth of MDA-MB-231 cells in triple-negative breast cancer by salinomycin combined with 17-AAG and its mechanism
}

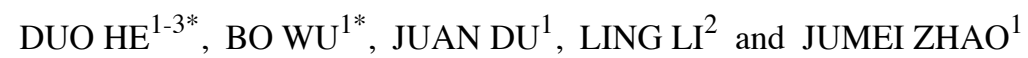 \\ ${ }^{1}$ Key Laboratory for Cancer Prevention and Treatment, Medical College of Yan'an University, \\ Yan'an, Shaanxi 716000; ${ }^{2}$ National Translational Science Center for Molecular Medicine, \\ Department of Cell Biology, School of Basic Medicine, The Fourth Military Medical University, Xi'an, \\ Shaanxi 710032; ${ }^{3}$ Department of Pathology, Yan'an People's Hospital, Yan'an, Shaanxi 716000, P.R. China
}

Received April 12, 2021; Accepted December 16, 2021

DOI: $10.3892 / \mathrm{ol} .2022 .13258$

\begin{abstract}
Salinomycin (SAL), a typical ion carrier antibiotic, inhibits tumor growth and metastasis by inducing apoptosis or autophagy in cancer or cancer stem cells and thus overcomes drug resistance. 17-allylamino-17-demethoxygeldanamycin (17-AAG), a heat shock protein Hsp90 competitive inhibitor, also has a role in inhibiting tumor development. However, their combination on the growth of breast cancer cells and its specific mechanism remains to be elucidated. The present study tested the influence of SAL and 17-AAG on cell growth, apoptosis and autophagy by MTT assays, Annexin V-FITC and propidium iodide double staining assay and immunoelectron microscopy. The influence of SAL and 17-AAG on proteomics was investigated by isobaric tag for relative and absolute quantitation. It was found that SAL combined with 17-AAG synergistically inhibited the cell growth and induced the apoptosis in a concentration-dependent manner, with the expression of caspase 3 and Bcl-2 were decreased while the expression of Bax was increased. In addition, SAL combined with 17-AAG inhibited autophagy, with the expression of microtubule-associated protein 1 light chain 3, Beclin1, p62 being decreased. Mechanistically, SAL combined with 17-AAG synergistically
\end{abstract}

Correspondence to: Dr Ling Li, National Translational Science Center for Molecular Medicine, Department of Cell Biology, School of Basic Medicine, The Fourth Military Medical University, 169 Changle West Road, Xi'an, Shaanxi 710032, P.R. China E-mail: liling25@fmmu.edu.cn

Dr Jumei Zhao, Key Laboratory for Cancer Prevention and Treatment, Medical College of Yan'an University, 580 Shrine Road, Yan'an, Shaanxi 716000, P.R. China

E-mail:jmz2003.stu@163.com

${ }^{*}$ Contributed equally

Key words: salinomycin, 17-AAG, apoptosis, autophagy, breast cancer cells inhibited the reactive oxygen species/JNK signaling pathway. In conclusion, SAL combined with $17-\mathrm{AAG}$ had a synergistic inhibitory effect on cell growth of breast cancer via inducing apoptosis and inhibiting autophagy. The present study might provide a new strategy for potential clinical application of SAL as a new anti-tumor drug especially as a drug combination with other molecular targeting therapeutics.

\section{Introduction}

Breast cancer is one of the common malignant tumors in women and the incidence rate ranks first among female malignant tumors (1). Triple-negative breast cancer (TNBC), a subtype of breast cancer with negative expression of estrogen receptor (ER), progesterone receptor (PR) and proto-oncogene human epidermal growth factor receptor 2 receptor (HER2), is resistant to endocrine and molecular targeted therapy and prone to local recurrence and distant metastasis (2). Chemotherapy is therefore the main treatment option for triple-negative breast cancer (3). However, patients with breast cancer are usually resistant to chemical therapeutics, which is the main reason of poor prognosis $(4,5)$. According to the theory of tumor stem cells, tumor stem cells serve an important role in the survival, proliferation, metastasis and recurrence of tumors. Thus, targeted killing of tumor stem cells may be the key to treat tumors $(6,7)$. Therefore, it is urgent to develop new chemotherapeutic drugs that can kill triple negative breast cancer rand study its specific mechanism, so as to find effective therapeutic targets.

Salinomycin (SAL), an ionophore antibiotic isolated from the fermentation broth of Streptomyces albicularis (strain no. 80614), kills pathogenic microorganisms by interfering with the cation $\left(\mathrm{Na}^{+}\right.$and $\left.\mathrm{K}^{+}\right)$balance inside or outside the cell and altering the osmotic pressure (8). Gupta et al (9) showed that SAL selectively exterminates breast cancer stem cells (CSCs), with an efficiency 100 times higher than that of paclitaxel. Since then, a number of in vivo and in vitro studies $(10,11)$ have demonstrated that SAL inhibits CSCs in various types of tumor (12). SAL helps tumor cells or CSCs overcome the apoptosis resistance caused by P53 
gene mutation, or $\mathrm{Bcl}-2$, 26S proteasome and $\mathrm{P}$-glycoprotein overexpression (13), increasing the DNA damage caused by oxidative stress (14) and inducing autophagy and subsequent apoptosis (15). In addition, SAL reverses drug resistance and increases the sensitivity of chemical drugs by inhibiting the activity of ATP binding box transporter superfamily and blocking the signaling pathway of $\mathrm{Wnt} / \beta$-catenin, $\mathrm{Akt} / \mathrm{NF}-\kappa \mathrm{B}$ and others (16-18). Therefore, SAL might be a potential clinically effective and highly selective anti-tumor drug. However, its severe neurotoxic and muscular toxicity cannot be ignored. To reduce the side effects of SAL, the development of combined treatment strategy or targeting delivery system is required.

17-allylamino-17-demethoxygeldanamycin (17-AAG) is an inhibitor of chaperone heat shock protein 90 (Hsp90), which binds to ATP-binding region of Hsp90, inhibits the formation of multiprotein complex comprising co-chaperone proteins and induces the degradation of client proteins through the ubiquitin-proteasome pathway (19). During the process of malignant transformation, Hsp90 stabilizes and protects mutated proteins from proteasomal degradation and enables the sustained survival and cell growth of cancer cells. By inhibiting Hsp90, 17-AAG has been widely investigated in preclinical and clinical research as a single agent or in combination with other anticancer agents for a wide range of types of human cancer. For example, 17-AAG inducing cell apoptosis by blocking the customer protein Hsp90 hypoxia-inducible factor $1 \alpha$ transcription function (20) and influencing the colony formation ability of CSCs and the growth of tumor cells (21). 17-AAG can directly interact with voltage-dependent anion channel through hydrophobic interaction, independent of Hsp90, to increase intracellular calcium ion concentration, prompting increased intracellular calcium ion concentration (22). In addition, 17-AAG can kill cancer cells through the synergistic action of the upstream molecules of CD95 death receptor with MAPK/ERK 1/2 inhibitors (23). In addition, 17-AAG can affect the stability of Akt, leading to the deletion and expression of Akt and enhancing the oxidative stress mediated by sulfhydryl in cancer cells, thus effectively increasing the sensitivity of cancer cells to chemotherapy (24).

In our previous study, SAL significantly inhibited the proliferation of breast cancer cells via reducing the expression of breast CSCs marker ALDH (25). To further enhance efficacy and reduce toxicity, the present study investigated the combined effect of SAL with 17-AAG on apoptosis and autophagy in breast cancer cells and the corresponding molecular mechanism. It may provide a theoretical basis for the potential combined treatment strategy involving SAL.

\section{Materials and methods}

Cell lines and cell culture. Human breast cancer cell line MDA-MB-231 was obtained from the American Type Culture Collection. The cells were incubated with RPMI-1640 medium (HyClone; Cytiva) containing 10\% fetal bovine serum (FBS; HyClone; Cytiva), $100 \mathrm{U} / \mathrm{ml}$ penicillin and $100 \mathrm{U} / \mathrm{ml}$ streptomycin and passaged at a ratio of 1:2 or 1:3. All cell lines were tested for mycoplasma and characterized by short tandem repeat profiling analysis (Cenvino).
Drugs. SAL was purchased from China Institute of Veterinary Drug Control, and 17-AAG was purchased from MedChemExpress. SAL and 17-AAG were dissolved in DMSO and administrated at the concentration of $1-32 \mu \mathrm{M}$ or $1.25 \mathrm{nM}$, respectively.

MTT assay. Single cell suspension with concentration of $5 \times 10^{4}$ cells $/ \mathrm{ml}$ was prepared from logarithmic growth stage cells. Briefly, $5 \times 10^{3}$ cells were seeded to each well of 96-well plates and were cultured overnight. Then, cells were treated with SAL alone $(1,2,4,8,16$ and $32 \mu \mathrm{M})$ or in combination with 17-AAG (1.25 nM) for 24, 48 and $72 \mathrm{~h}$. Next, cells were incubated with $20 \mu \mathrm{l}$ MTT ( $5 \mathrm{mg} / \mathrm{ml}$; Sigma; cat. no. M2003) for $4 \mathrm{~h}$, followed by $150 \mu \mathrm{l}$ DMSO for dissolving the crystals. The optical density values were detected at $490 \mathrm{~nm}$ and the relative cell growth was calculated and expressed as $\mathrm{IC}_{50}$ values using GraphPad Prism 6.0 (GraphPad Software, Inc.). Untreated cells were chosen as a control, which were cells incubated with DMSO (solvent for SAL) and DMSO (solvent for 17-AAG). Combination index (CI) was calculated by the CompuSyn software (version 1.0; Biosoft) using the Chou-Talalay method: $\mathrm{CI}=1$, additive effect; $\mathrm{CI}<1$, synergistic effect; CI >1, antagonistic effect $(26,27)$.

Apoptosis assay. Cells were treated with SAL and/or 17-AAG for $48 \mathrm{~h}$. Then, $5 \times 10^{5}$ cells were labeled with the Annexin V and propidium iodide (PI) using FITC Annexin V Apoptosis Detection kit with PI (BioLegend, Inc, cat. no. 641904) according to the manufacturer's protocol. The labeled cells were immediately measured with a BD FACSCalibur Flow Cytometer (BD Biosciences) and quantified with CellQuest software (version 5.1; BD Biosciences) for early and late apoptotic cells.

$R T-q P C R$. Total RNA was extracted from $5 \times 10^{5}$ cells treated with SAL and/or 17-AAG treated cells for $48 \mathrm{~h}$ using TRIzol ${ }^{\circledR}$ (Thermo Fisher Scientific, Inc.), and the quantity and purity of RNA were detected using NanoDrop 1000 spectrophotometer (Thermo Fisher Scientific, Inc.) according to the manufacturer's instructions. Total RNAs $(1 \mu \mathrm{g})$ was reversely transcribed into cDNA by Super Script first-strand synthesis system (Invitrogen; Thermo Fisher Scientific, Inc.) as described previously (9). Prepared cDNA was then subjected to quantitative PCR analysis using the Strata gene Mx3005P Multiplex quantitative PCR system (Agilent Technologies, Inc.) with $2 X$ SYBR Green qPCR Master Mix (Bimake; cat. no. B21203). A total of $20 \mu \mathrm{l}$ real-time fluorescence PCR reaction mixture was used and the reaction conditions were as follows: Pre-denaturation at $95^{\circ} \mathrm{C}$ for $5 \mathrm{~min}$, reaction at $95^{\circ} \mathrm{C}$ for $20 \mathrm{sec}, 58^{\circ} \mathrm{C}$ for $20 \mathrm{sec}, 72^{\circ} \mathrm{C}$ for $20 \mathrm{sec}$ and $72^{\circ} \mathrm{C}$ for $10 \mathrm{~min}$ for a total of 40 cycles. The relative expression of genes was analyzed by the comparative $\mathrm{Ct}$ method. The data are presented as the fold change, which was calculated as $2^{-\Delta \Delta \mathrm{Cq}}(\Delta \Delta \mathrm{Cq}=\Delta \mathrm{C}$ $\left.\mathrm{q}_{\text {treated }}-\Delta \mathrm{Cq}_{\text {control }}\right)(28) . \mathrm{Cq}$ is the cycle number at which fluorescence first exceeds the threshold. The $\Delta \mathrm{Cq}$ values from each target gene were obtained by subtracting the GAPDH Cq from the sample Cq. The experiment was repeated three times. The primer sequences are shown in Table I.

Western blotting. SAL and/or 17-AAG treated cells were collected and the total protein was extracted using the 
Table I. Primer sequences.

\begin{tabular}{lll}
\hline Gene & Orientation & Primer sequence (5'-3') \\
\hline Bcl-2 & Forward & GGTGGGGTCATGTGTGTGG \\
& Reverse & CGGTTCAGGTACTCAGTCATCC \\
Bax & Forward & CCCGAGAGGTCTTTTCCGAG \\
& Reverse & CCAGCCCATGATGGTTCTGAT \\
Caspase 3 & Forward & CATGGAAGCGAATCAATGGACT \\
& Reverse & CTGTACCAGACCGAGATGTCA \\
LC3 & Forward & AACATGAGCGAGTTGGTCAAG \\
& Reverse & GCTCGTAGATGTCCGCGAT \\
Beclin1 & Forward & CCATGCAGGTGAGCTTCGT \\
& Reverse & GAATCTGCGAGAGACACCATC \\
P62 & Forward & GCACCCCAATGTGATCTGC \\
& Reverse & CGCTACACAAGTCGTAGTCTGG \\
GAPDH & Forward & GGAGCGAGATCCCTCCAAAAT \\
& Reverse & GGCTGTTGTCATACTTCTCATGG
\end{tabular}

phospho-RIPA buffer (1 M Tris- $\mathrm{HCl}$ at $\mathrm{pH} 7.5,5 \mathrm{M} \mathrm{NaCl}$, $0.01 \%$ NP-40, 0.5 M EGTA and 10\% SDS) supplemented with a complete EDTA-free protease inhibitor cocktail (Roche Diagnostics). Protein was quantitated by a bicinchoninic acid kit (Abcam), separated in SDS-PAGE gels (8 or 10\%) with protein loaded of $10 \mu \mathrm{l}$ in per lane and electrically transferred onto polyvinylidene fluoride membranes (MilliporeSigma) membrane. Following blocking in PBS $+5 \%$ bovine serum albumin (Abcam) at room temperature, the membranes were then incubated with the following primary antibodies: Cleaved-caspase 3 (1:500; ProteinTech Group, Inc.; cat. no. 25546-1-AP), Bcl-2 (1:1,000; Cell Signaling Technology, Inc.; cat. no. 15071S), Bax (1:1,000; Cell Signaling Technology, Inc.; cat. no. 89477), microtubule-associated protein 1 light chain 3 (LC3)B (1:1,000; Abcam; cat. no. ab51520), Beclin1 (1:1,000; Abcam; cat. no. ab210498), P62 (1:1,000; Abcam; cat. no. ab56416), JNK (1:1,000; Abcam; cat. no. ab76125) and phosphorylated (p-) JNK (1:1,000; Cell Signaling Technology, Inc.; cat. no. $9255 \mathrm{~S})$ at $4^{\circ} \mathrm{C}$ overnight; and subsequently with the Rabbit Anti-Mouse IgG H\&L (HRP) (1:5,000; Abcam; cat. no. ab6728) or Goat Anti-Rabbit IgG H\&L (HRP) (1:5,000; Abcam; cat. no. ab6721) at room temperature for $1 \mathrm{~h}$. The protein signals were developed using the Enlight Western blot ECL reagents (Engreen Biosystem Co., Ltd.). GAPDH (1:2,500; Abcam; cat. no. ab9485) or $\alpha$-tubulin (1:5,000; Abcam; cat. no. ab7292) was used as a loading control.

Transmission electron microscopy. Cells were collected $48 \mathrm{~h}$ after treatment with SAL alone and in combination with 17-AAG. The cell concentration was adjusted to $4 \times 10^{6}-1 \times 10^{7}$ cells $/ \mathrm{ml}$ Cells were fixed with $2.5 \%$ glutaraldehyde buffered in $0.1 \mathrm{M}$ sodium cacodylate $(\mathrm{pH} 7.4)$ at $5^{\circ} \mathrm{C}$ for $\sim 4 \mathrm{~h}$ on ice, then rinsed in sodium cacodylate buffer and post-fixed in $1 \%$ aqueous osmium tetroxide (buffered in $0.1 \mathrm{M}$ sodium cacodylate) at $5^{\circ} \mathrm{C}$ for $\sim 2 \mathrm{~h}$ and then rinsed and stored in the buffer at $4^{\circ} \mathrm{C}$. Cells were later dehydrated in an acetone/ethanol series and transferred to propyleneoxide and then subsequently embedded in Glycidether 100 (formerly
Epon) (Abcam; cat. no. c5318). Following polymerization, semi-thin sections $(1 \mu \mathrm{m})$ and ultrathin sections $(60-90 \mathrm{~nm})$ were cut and stained with toluidine blue for 20-30 min at room temperature. Digital micrographs were captured with a JEOL JEM1010 electron microscope (JEOL, Ltd. Tokyo:6951).

Tandem Mass Tag (TMT) quantification for proteomics. Cells were collected $48 \mathrm{~h}$ after treatment with SAL alone and in combination with 17-AAG. The cells were lysed by adding five cell-pellet volumes of lysis buffer (100 $\mu \mathrm{l}$ of Lysis Buffer for a $20 \mu \mathrm{l}$ cell pellet) according to the TMT labelling kit instructions (Thermo Fisher Scientific, Inc). Peptides were labelled with the TMT Iso baric Mass Tags (Thermo Fisher Scientific, Inc.); the lysate was centrifuged at $16,000 \mathrm{x} \mathrm{g}$ for $10 \mathrm{~min}$ at $4^{\circ} \mathrm{C}$, and adjusted to a final volume of $1,000 \mu 1$ with $100 \mathrm{nM}$ TEAB. After adding $5 \mu \mathrm{l}$ TCEP $(200 \mathrm{nM})$ and incubating at $55^{\circ} \mathrm{C}$ for $1 \mathrm{~h}$, TEAB was diluted away from light in $132 \mu \mathrm{l}$ iodoacetamide to a concentration $375 \mathrm{nM}$. Next, 6 volumes of pre-chilled acetone $\left(-20^{\circ} \mathrm{C}\right)$ and $20 \mu \mathrm{l}$ trypsin storage solution (room temperature) were added and incubated for $5 \mathrm{~min}$. Lastly, $2.5 \mu \mathrm{l}$ trypsin per $100 \mu \mathrm{g}$ of protein was added to digest the sample overnight at $37^{\circ} \mathrm{C}$. Finally, Proteome Discoverer (version 2.1; Thermo Fisher Scientific, Inc.) was used to analyze data, screen differential proteins.

Mito-ROS. Cells were treated with SAL and/or 17-AAG for $48 \mathrm{~h}$ at $37^{\circ} \mathrm{C}$. The concentration of cell suspension was adjusted to $5 \times 10^{5}$ cells $/ \mathrm{ml}$. The cell suspension was transferred to an Eppendorf test tube, centrifuged at 1,000 x $\mathrm{g}$ for $5 \mathrm{~min}$ at room temperature and the supernatant discarded. The blank control group and experimental group were set up. The Mito Tracker Red CMX Ros using Mito Tracker Red CMX Ros-Special Packaging (Thermo Fisher Scientific, Inc.; cat. no. M7512) was diluted in serum-free medium to a final concentration of $1 \mathrm{mM}$ according to the manufacturer's protocol and incubated at $37^{\circ} \mathrm{C}$ for $15-35 \mathrm{~min}$ away from light and mixed every $5 \mathrm{~min}$. Following incubation, the centrifuged at $1,000 \mathrm{x} \mathrm{g}$ for $5 \mathrm{~min}$ and the supernatant discarded. Following washing with PBS three times, the cells were suspended with $500 \mu 1$ PBS and placed on ice for flow cytometry detection. Mito-tracker Red CMXRos fluoresce in red with a maximum excitation wavelength of $579 \mathrm{~nm}$ and a maximum emission wavelength of $599 \mathrm{~nm}$. A FACSCalibur flow cytometer (BD Biosciences) was used to detect the fluorescence intensity of different groups of cells at specific wavelengths to compare the ROS content in different groups of mitochondria using FlowJo (version 10; BD Biosciences).

Statistical analysis. All data are obtained from three replicate experiments and expressed as mean \pm standard deviation of three independent experiments. GraphPad Prism 6.0 software (GraphPad Software, Inc.) was used for statistical analysis and graph rendering. One-way ANOVA followed by Tukey's test was used for comparing continuous variables multiple groups. $\mathrm{P}<0.05$ was considered to indicate a statistically significant difference. Kyoto Encyclopedia of Genes and Genomes (KEGG) pathway analysis was carried out on the proteomics data. In the KEGG database (https://www.kegg.jp/), KO (KEGG Orthology) is a classification system for genes and their products. Lineal homologous genes with similar 
A

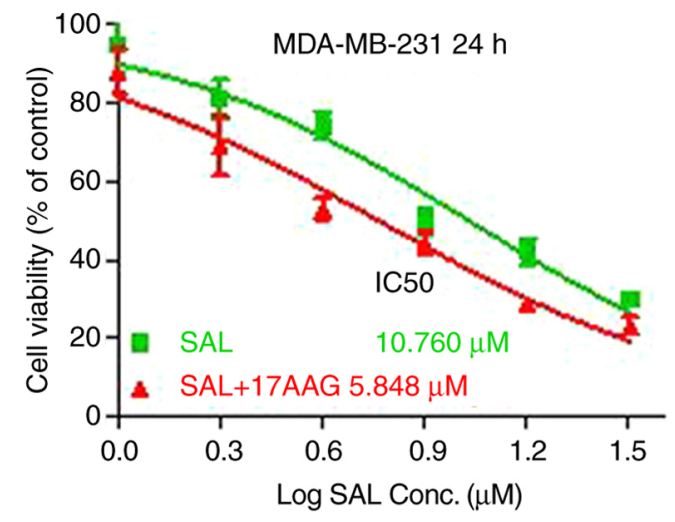

C

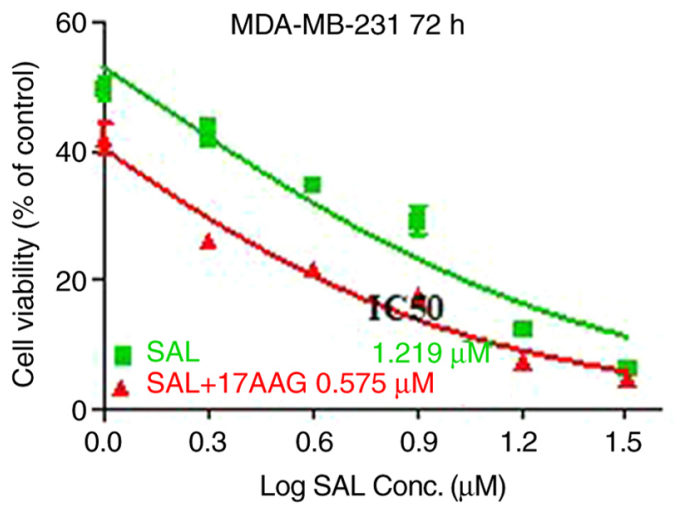

E

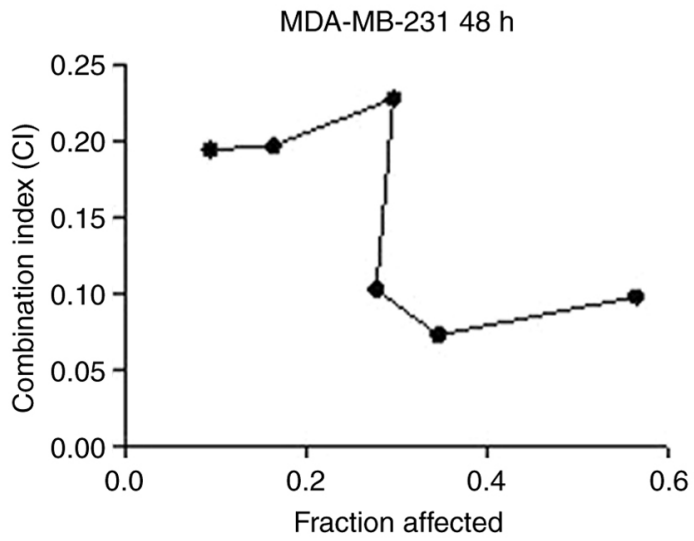

B

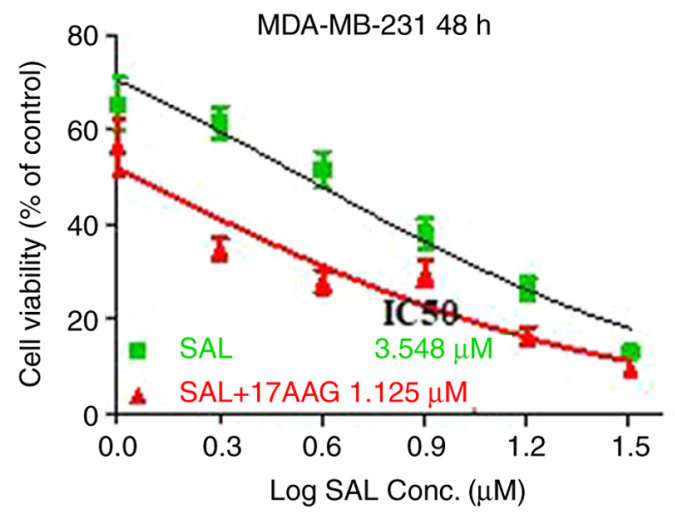

D

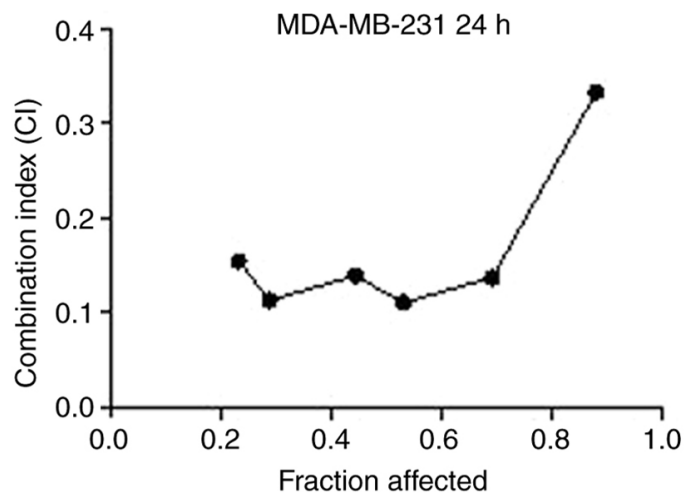

F

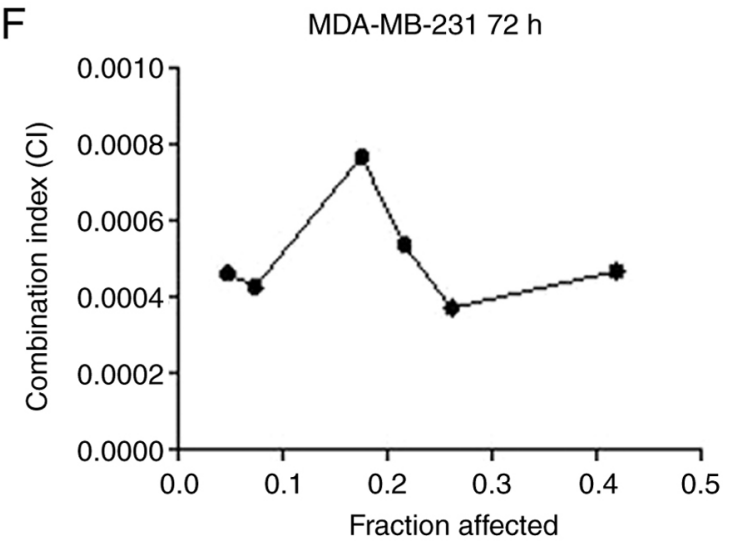

Figure 1. Combination of SAL with 17-AAG synergistically inhibits cell growth in human breast cancer cells. Relative cell growth in human breast cancer cells treated with different concentrations of SAL alone or in combination with 17AAG for (A) 24 , (B) 48 and (C) 72 h, respectively. IC 50 values was calculated using GraphPad Prism 6.0. The solvent treated cells were chosen as a control. Synergistic growth inhibitory effects of SAL and 17AAG on human breast cancer cells for (D) 24, (E) 48 and (F) 72 h, respectively. CI was calculated by isobologram analysis using the Chou-Talalay method. Data represented are from three independent experiments. SAL, salinomycin; 17-AAG, 17-allylamino-17-demethoxygeldanamycin; CI, combination index, Conc., concentration.

functions on the same pathway and their products are grouped together and assigned the same KO label (29). To annotate KEGG pathways in the target protein set, KEGG Orthology and Links Annotation (KOALA) (30) was used to classify the target proteins by KO the KEGG database (http://www.kegg. jp/kegg-bin/show_pathway?ko04010+K04440). The pathway information associated with the target protein was obtained automatically according to KO classification. For KEGG pathway enrichment analysis, Fisher's Exact Test was used to screen the proteins associated with specific KEGG pathway and analyse their relative enrichment. Pathways with a false discovery rate $<0.01$ were considered significantly enriched.

\section{Results}

Combination of SAL and 17-AAG synergistically inhibits cell growth in human breast cancer cells. To explore the possible Synergistic inhibitory effect of SAL and 17-AAG, the MTT assay was used to detect the relative cell growth in human breast cancer cells treated with SAL alone or in combination with 17-AAG. The results showed that SAL alone significantly reduced cell growth in a dose- and time-dependent manner, with $\mathrm{IC}_{50}$ of SAL being $10.760,3.548$ and $1.219 \mu \mathrm{M}$ in MDA-MB-231 cells for 24, 48 and $72 \mathrm{~h}$, respectively (Fig. 1A-C). In addition, the combination of 17-AAG with 
MDA-MB-231 Apoptosis ratio (\%)
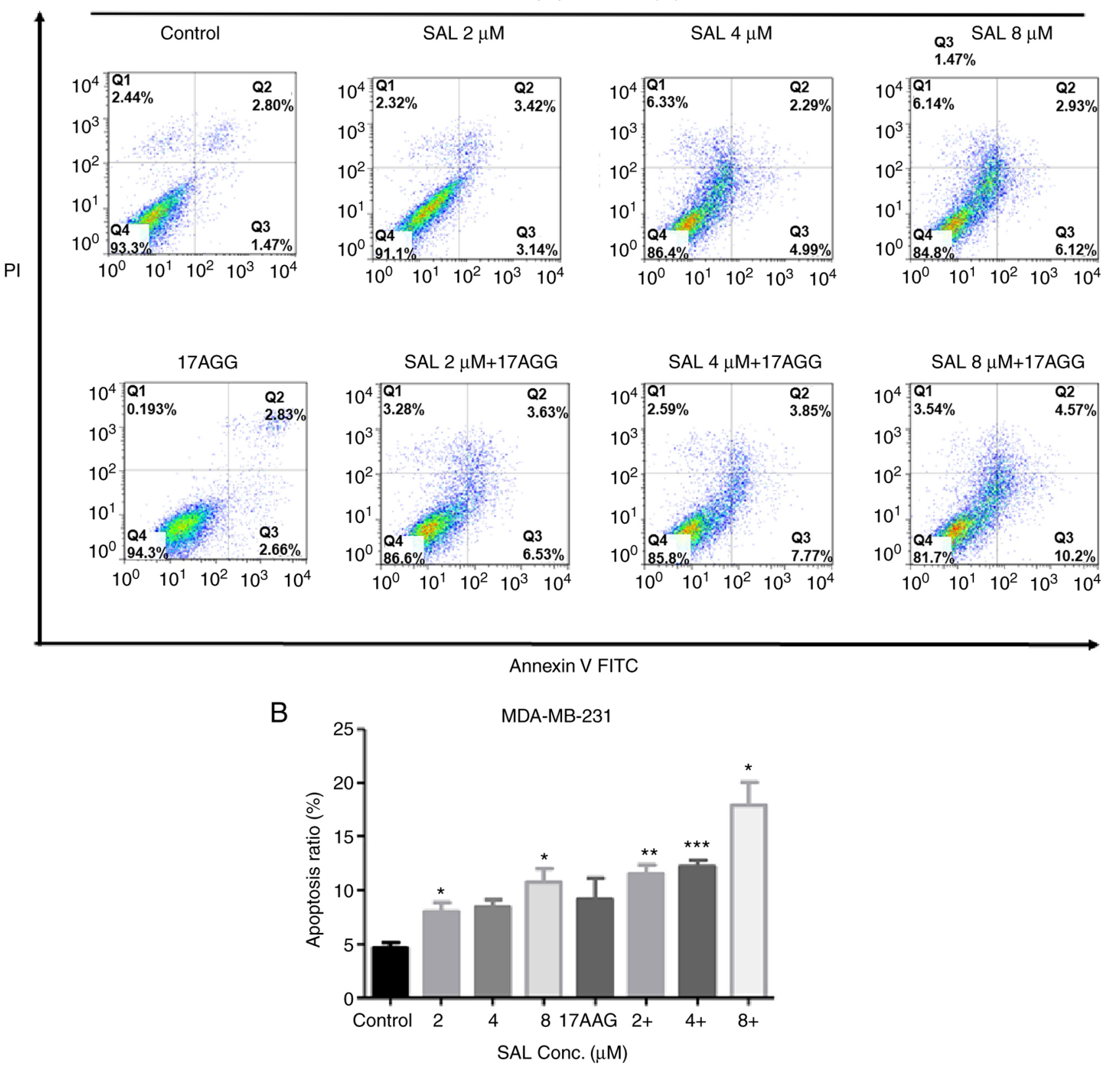

Figure 2. Combination of SAL and 17-AAG synergistically induced apoptosis in human breast cancer cells. (A) Apoptosis rate of breast cancer MDA-MB-231 with SAL alone and in combination with $17-A A G$ for $48 \mathrm{~h}$. (B) Statistics of apoptosis rate. ${ }^{*} \mathrm{P}<0.05,{ }^{* *} \mathrm{P}<0.01,{ }^{* * * *} \mathrm{P}<0.001$ vs. control group. SAL, salinomycin; 17-AAG, 17-allylamino-17-demethoxygeldanamycin; PI, propidium iodide; Conc., concentration.

SAL clearly decreased $\mathrm{IC}_{50}$ of SAL by $50 \%$ compared with that in cells treated with SAL alone, with the highest reduction occurring at $48 \mathrm{~h}$. These results indicated that the combination treatment of SAL and 17-AAG was more effective in inhibiting cell growth when compared with the single treatment of SAL, which implying an interaction between SAL and 17-AAG.

To further clarify the combined growth inhibitory effect of SAL and 17-AAG on MDA-MB-231 cells, the Chou-Talalay combined index method was used. The results showed that the growth inhibition ratio of SAL combined with 17-AAG in MDA-MB-231 cells for $24 \mathrm{~h}$ was $0.8807773,0.6917017$, $0.5309874,0.4438025,0.2883403$ and 0.2326681 and the corresponding combination index (CI) was 0.33311, 0.13707, $0.11066,0.13916,0.11332$ and 0.15435 . As CI values $<1$ indicate synergism in drug combinations, the data suggested the combination of SAL and 17-AAG had a synergistic effect on the cell growth of breast cancer cells (Fig. 1D-F). With time, CI was significantly increased, indicating that the synergistic inhibition between SAL and 17-AAG occurred in time-dependent manner.

Combination of SAL and 17-AAG synergistically induces apoptosis in human breast cancer cells. Synergism of anticancer drug can be occurred from the combination with different mechanisms and/or modes of actions. SAL and 17-AAG was reported to have a role in inducing apoptosis, thus the apoptotic ratios in human breast cancer cells treated with SAL and/or 17-AAG was first detected. As shown in Fig. 2, 


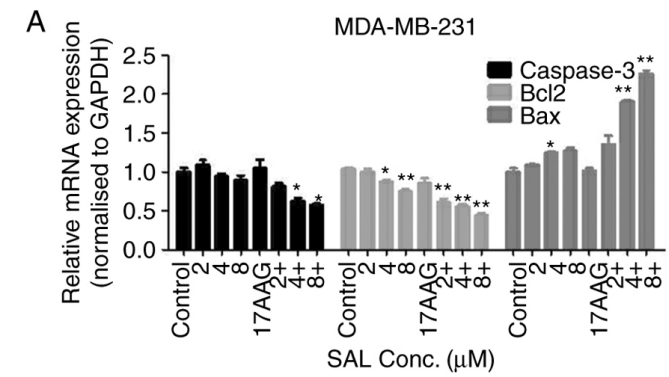

B

\begin{tabular}{rllllllll} 
& \multicolumn{1}{c}{ MDA-MB-231 } \\
\cline { 2 - 5 } SAL $(\mu M)$ & - & 2 & 4 & 8 & - & 2 & 4 & 8 \\
17AAG $(n M)$ & - & - & - & - & + & + & + & +
\end{tabular}
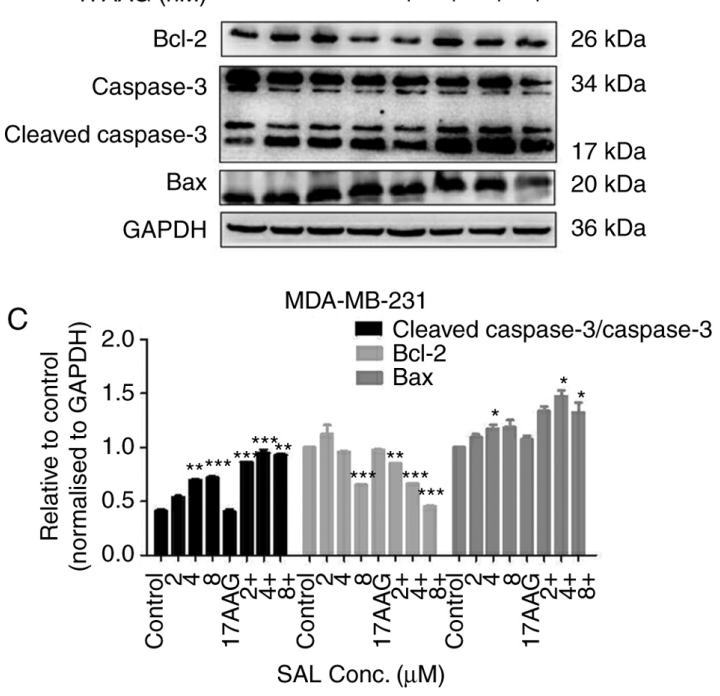

Figure 3. Expression of apoptosis-related genes in human breast cancer cells. (A) mRNA expression of Bcl-2, Bax and caspase 3 in MDA-MB-231 breast cancer cells from SAL treated with SAL alone and combined with 17-AAG. (B) Protein expression of Bcl-2, Bax and caspase 3 in MDA-MB-231 cells of breast cancer from SAL treated with SAL alone and combined with 17-AAG. (C) Quantitative analysis. ${ }^{*} \mathrm{P}<0.05,{ }^{* *} \mathrm{P}<0.01,{ }^{* * * *} \mathrm{P}<0.001$ vs. control group. SAL, salinomycin; 17-AAG, 17-allylamino-17-demethoxygeldanamycin; Conc., concentration.

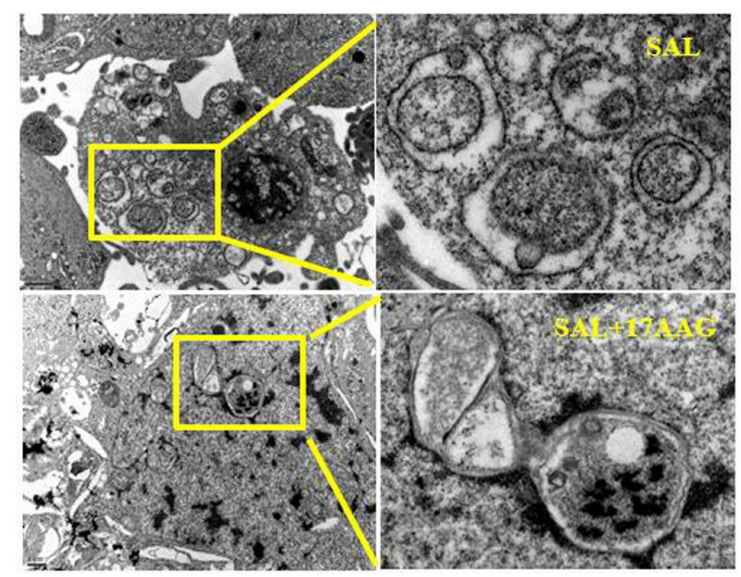

Figure 4. Formation of autophagosome and morphological changes of cell apoptosis under electron microscope. Magnification, x30,000). Effects of SAL and 17AAG alone and in combination on autophagosomes and apoptosis of human breast cancer MDA-MB-231 cells.

the apoptotic ratio increased in a dose-dependently manner in SAL treated cells. The SAL-induced apoptotic ratios were further significantly enhanced when SAL and 17-AAG

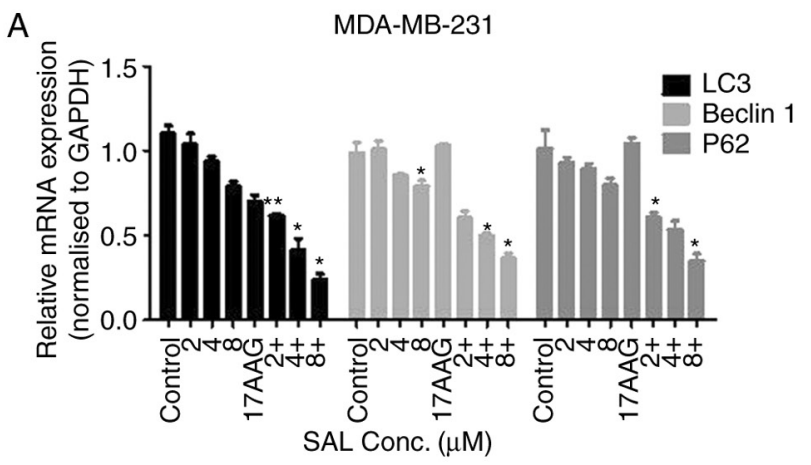

$\mathrm{B}$
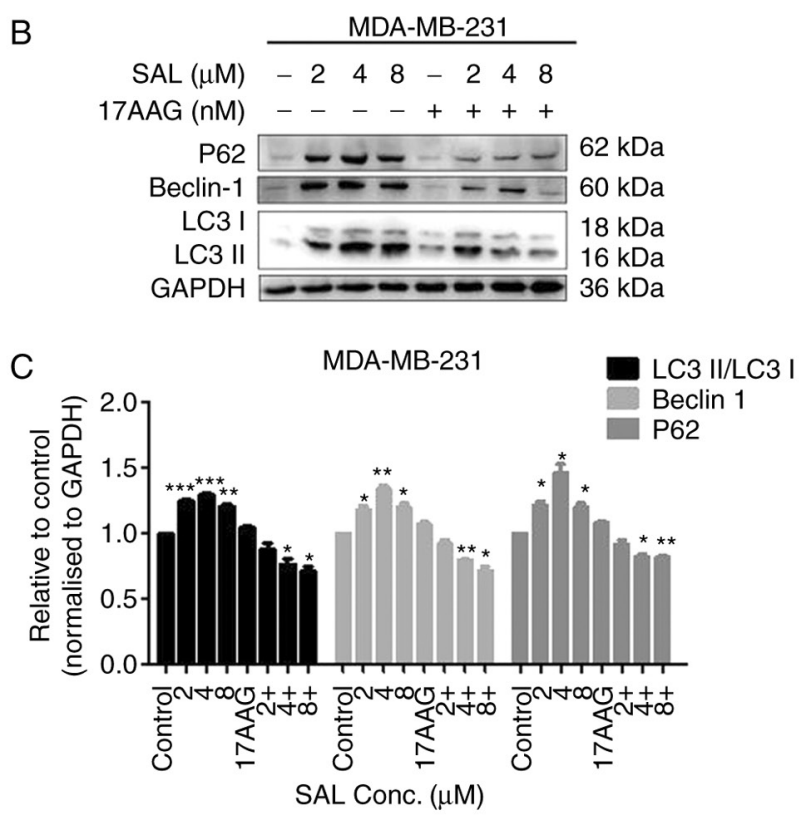

Figure 5. SAL and 17-AAG combination synergistically inhibits autophagy in human breast cancer cells. (A) Expression levels of LC3, Beclin1 and P62 mRNA. (B) Effects of LC3, Beclin1 and P62 protein expression levels. (C) Quantitative analysis. ${ }^{*} \mathrm{P}<0.05,{ }^{* *} \mathrm{P}<0.01,{ }^{* * *} \mathrm{P}<0.001$ vs. control group. SAL, salinomycin; 17-AAG, 17-allylamino-17-demethoxygeldanamycin; LC3, microtubule-associated protein 1 light chain 3; Conc., concentration.

were combined. Correspondingly, the mRNA and protein expression of genes involving in apoptosis signaling pathway, such as Bcl-2 and caspase-3, in SAL treated cells were decreased, while the mRNA and protein expression of Bax and cleaved caspase- 3 was increased, as compared with the cells treated with combined SAL and 17-AAG (Fig. 3). The results showed that SAL in combination with 17-AAG greatly activated the apoptosis-related pathways and then promoted apoptosis in breast cancer cells.

Combination of SAL and 17-AAG synergistically inhibits autophagy in human breast cancer cells. Transmission electron microscopy was used to observe the effect of SAL alone and in combination with 17-AAG on MDA-MB-231 cell apoptosis and autophagy. The results showed that SAL alone and in combination with 17-AAG could induce apoptosis and autophagy in human breast cancer MDA-MB-231 cells. Under the microscope, the formation of autophagic bodies, swelling and deformation of mitochondria, as well as obvious nuclear fragmentation and vacuoles in the cytoplasm, could be observed, suggesting the occurrence of apoptosis and 
A

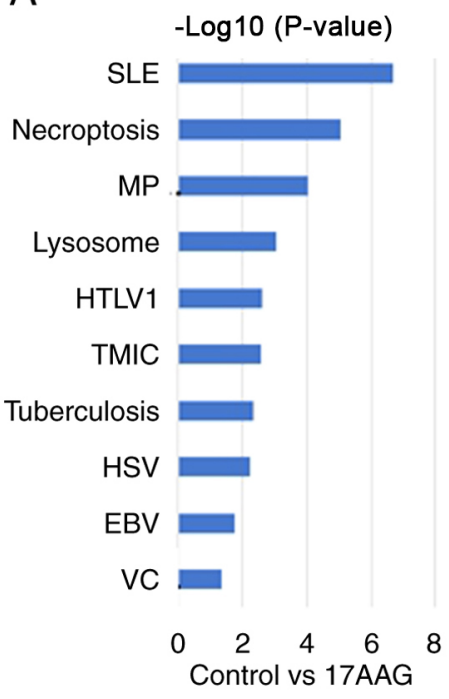

B

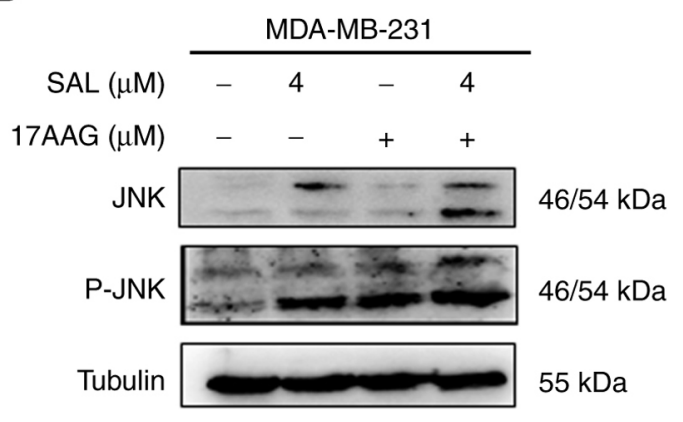

D

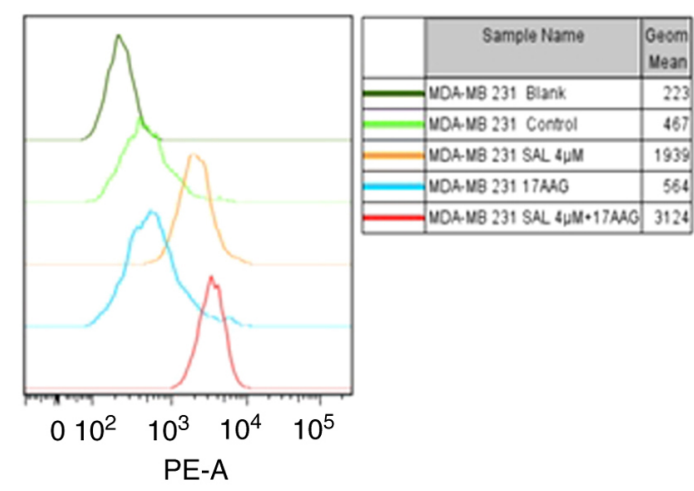

-Log10 (P-value)

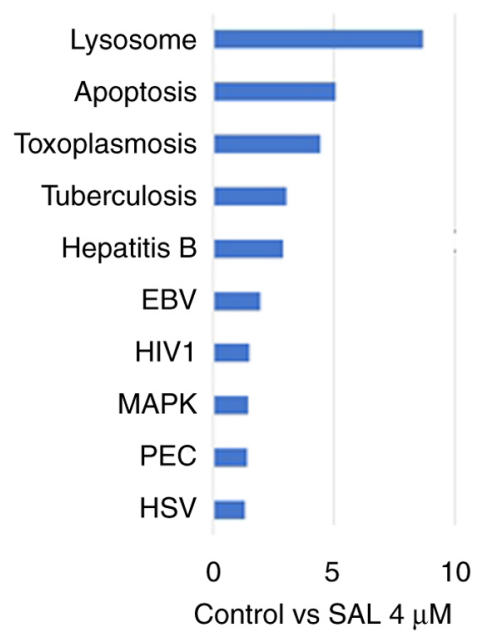

-Log10 (P-value)

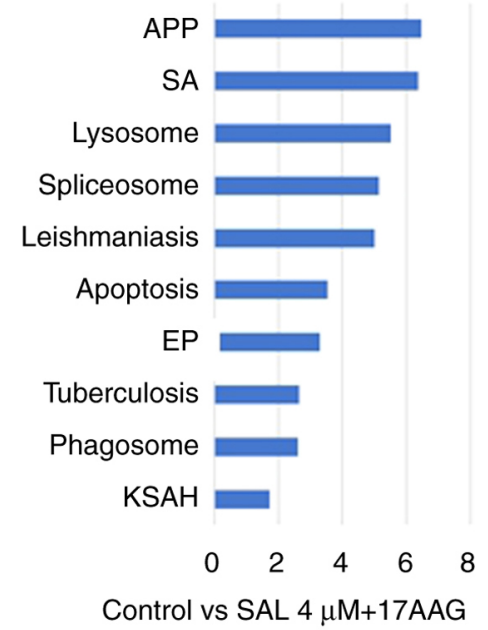

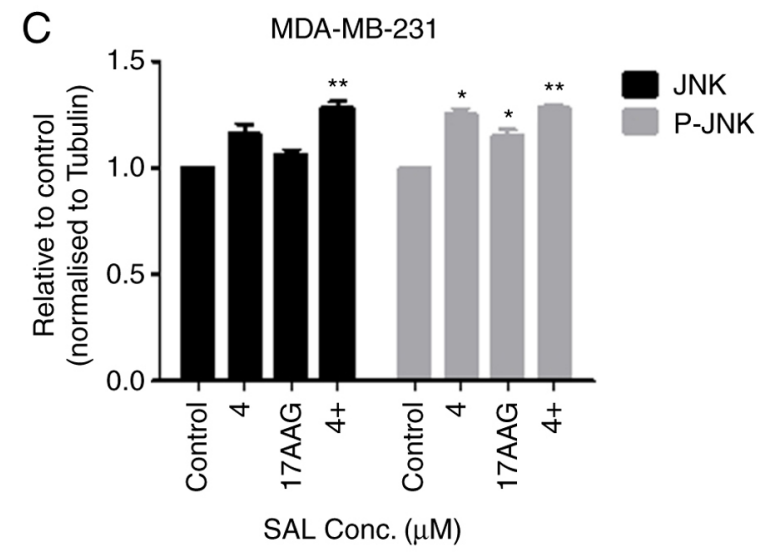

E

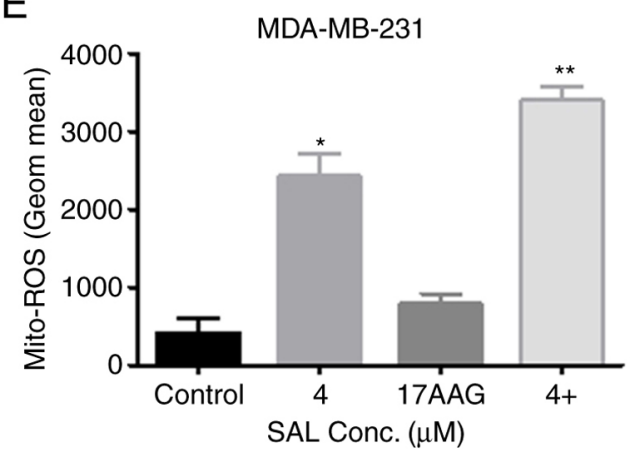

Figure 6. Combination of SAL and 17-AAG synergistically induced apoptosis and inhibited autophagy through the ROS-JNK signaling pathway in human breast cancer cells. (A) Kyoto Encyclopedia of Genes and Genomes pathway statistics with significant enrichment (top 10). (B) Effects of JNK and p-JNK protein expression levels. (C) Effect of ROS content in cells. condition for $48 \mathrm{~h}$. (D) Quantitative statistics of protein. (E) Peak statistics of Mito-Ros. ${ }^{*} \mathrm{P}<0.05,{ }^{* *} \mathrm{P}<0.01$ vs. control group. SAL, salinomycin; 17-AAG, 17-allylamino-17-demethoxygeldanamycin; ROS, reactive oxygen species; p-, phosphorylated; SLE, systemic lupus erythematosus; MP, metabolic pathways; HTLV1, human T-cell lymphotropic virus 1; TMIC, transcriptional misregulation in cancer; HSV, herpes simplex virus; EB, Epstein-Barr virus; VC, viral carcinogenesis, HIV1, human immunodeficiency virus 1; PEC, pathogenic Escherichia coli infection; APP, antigen processing and presentation; SA, Staphylococcus aureus; EP, estrogen pathway; KSAH, Kaposi sarcoma-associated herpesvirus; Conc., concentration.

autophagy (Fig. 4). Compared with the single drug group, the autophagosomes in the combined drug group were reduced and the cells in the combined drug group were mainly apoptotic, with obvious nuclear fragmentation observed under the microscope. As compared with the single drug group, apoptosis increased and autophagy decreased in the combination group. It was suggested that the combination of SAL and 17-AAG may induce apoptosis and inhibit autophagy, thereby affecting the growth of MDA-MB-231 cells in triple-negative breast cancer. 
SAL was used alone $(2,4$ and $8 \mu \mathrm{M})$ or in combination with 17-AAG $(1.25 \mathrm{nM})$ on breast cancer MDA-MB-231 cells for $48 \mathrm{~h}$. The protein and mRNA expression levels of LC3, Beclin1 and P62 were all lower than those of the control group and the protein bands became lighter and narrower. Compared with the single drug group, the histone and mRNA expression levels of the combined drug group were more significantly reduced (Fig. 5).

Combination of SAL and 17-AAG synergistically induces apoptosis and inhibits autophagy through the reactive oxygen species (ROS)-JNK signaling pathway in human breast cancer cells. TMT results showed that SAL alone and in combination with 17-AAG can cause changes in apoptosis and autophagy pathways (Fig. 6A). Furthermore, it was found that SAL combined with 17-AAG caused significant changes in the MAPK signaling pathway proteins, with JNK and p-JNK proteins significantly upregulated. Therefore, the combination of SAL and 17-AAG may induce apoptosis and autophagy through the JNK signaling pathway and eventually affect the growth of breast cancer MDA-MB-231 cells. The proteomic results were further verified to investigate whether the mechanism of SAL and 17-AAG inhibiting the growth of breast cancer cells through apoptosis and autophagy was associated with the JNK signaling pathway. The protein expression of JNK and p-JNK in the MAPK pathway were detected by western blotting (Fig. 6B and C). The results showed that after breast cancer cells were treated with SAL alone and in combination with 17-AAG, the upregulation of JNK and $\mathrm{p}-\mathrm{JNK}$ proteins was observed. The expression of proteins in the combination group was higher than that in the single drug group. It was also found that SAL alone and combined with 17-AAG can synergistically induce the production of ROS in MDA-MB-231 cells and the ROS content in the combined group was higher than that in the single group (Fig. 6D and E), suggesting that SAL and 17-AAG may further activate the JNK pathway through ROS to further induce apoptosis and autophagy and influence the growth of breast cancer MDA-MB-231 cells.

\section{Discussion}

In 2012, Verdoodt et al (31) first discovered that SAL can activate autophagy after acting on colon and breast cancer cell lines. Subsequent studies showed that SAL could affect breast cancer cell proliferation by inducing apoptosis and autophagy $(18,31)$. However, the cytotoxicity of SAL is also a problem that cannot be ignored, which is the key factor affecting its curative effect. Therefore, how to enhance the selective killing effect of SAL between cancer or CSCs cells and normal cells and improve its therapeutic effect has become an important issue that requires urgent attention.

Combined drug use refers to the simultaneous or continuous use of two or more drugs to improve the efficacy and reduce the possibility of drug resistance without increasing toxicity, so as to achieve therapeutic effects. Through a literature review, it was found that SAL and 17-AAG overlap in certain anti-tumor mechanisms. The two drugs can affect the growth of cancer cells by inducing apoptosis, targeting CSCs and increasing oxidative stress, as well as affecting drug resistance in cancer cells. Therefore, the combined application of SAL

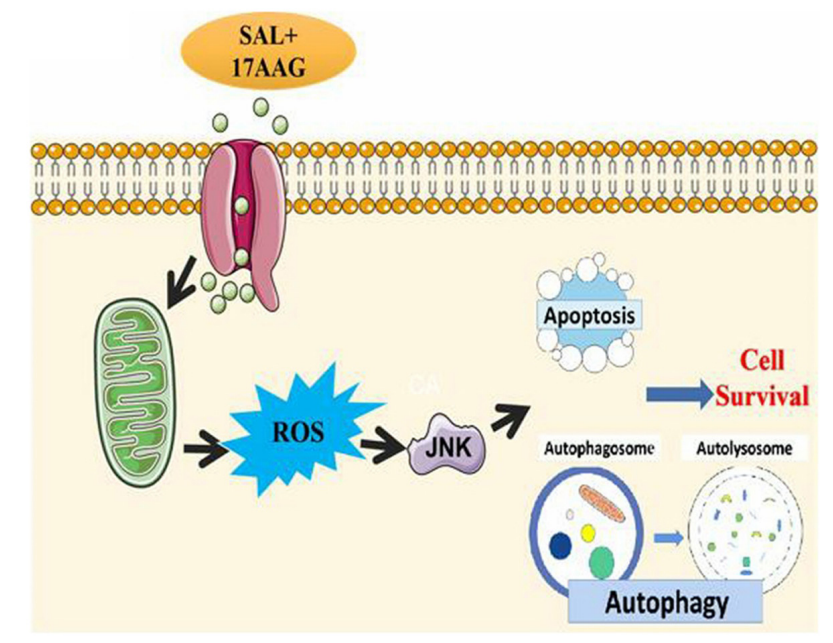

Figure 7. Mechanistic diagram of SAL combined with 17-AAG. SAL, salinomycin; 17-AAG, 17-allylamino-17-demethoxygeldanamycin; ROS, reactive oxygen species.

and 17-AAG may reduce the dose of SAL, thus reducing its cytotoxicity and effectively killing cancer cells or CSCs while reducing the occurrence of drug tolerance. The present study also showed that the use of SAL and 17-AAG alone and in combination can induce apoptosis, leading to an increase in the apoptotic ratio and upregulation of apoptosis-related protein expression, as well as the downregulation of autophagy-related protein expression in breast cancer cells, inhibition of protein expression and reduction of autophagosomes. The results also indicated that the combination of SAL and 17-AAG can affect breast cancer cell growth by inducing apoptosis and autophagy. The proteomic results showed that both SAL and 17-AAG alone and in combination could induce apoptosis and the autophagy signaling pathway, as well as inflict significant changes in the MAPK signaling pathway.

In addition, studies $(32,33)$ have shown that there is an association between autophagy and ROS. The inhibition of autophagy through autophagy inhibitors can significantly increase the level of ROS, and ROS elimination can significantly induce cell death. Studies have also shown that autophagy can promote cell apoptosis and ROS are considered to be the main molecules associated with cell apoptosis and autophagy $(34,35)$. ROS can regulate cell growth and survival, as well as inhibit the PI3K/Akt signaling pathway (36) and activate the MAPK signaling pathway (37). The present study found that SAL alone and in combination with 17-AAG could cause an increase in the ROS content. In cancer treatment, ROS are not only associated with autophagy, but are also a key factor affecting cell apoptosis and proliferation (38).

ROS can activate JNK through bispecific kinase JNKK and the activated JNK can promote the expression of pro-apoptotic proteins such as P53, Bax, Fas-ligand (FasL) and TNF, through transcription factor AP-1 (39). High expression of pro-apoptotic proteins, such as Bax and Bak, can promote the release of cytochrome $\mathrm{c}$ into the cytoplasm. The binding of cytochrome c and caspase-9 can activate caspase-3 (40). The activated caspase- 3 serves a very important role in that it can lyse autophagy-related proteins, which can enter the mitochondria, promote the release of cytochrome $\mathrm{c}$ and further promote 
the occurrence of cell apoptosis (41). Studies have shown that Beclin 1 can be cleaved by caspase- 3 to produce C-terminal fragment of Beclin 1, which can enter the mitochondria to promote the release of cytochrome c, inhibit autophagy and induce apoptosis $(42,43)$. In addition, expressed ligands, such as FasL and TNF, bind to the death receptor on the cell membrane to form a death-inducing signaling complex, which promotes the cleavage of the precursor caspase- 8 to generate the activated caspase- 8 (44). On the one hand, caspase- 8 can activate the downstream caspase- 8 of cell apoptosis to initiate the apoptosis signal and on the other hand, it can induce the production of cellular FLICE-inhibitory protein, viral FLICE-inhibitory protein and other substances that bind to Atg3. Thus, the binding of Atg3 and LC3 is inhibited, as is the occurrence of autophagy (45).

However, there are some limitations of the present study. First, it only focused on the synergistic inhibition effects on the MDA-MB-231 cell line, which is not enough to judge the efficacy of combined drugs and further application in human breast cancer. Other triple-negative breast cancer cell line should be used to verify the effect of this combination therapy in a future study. Second, the present study only explored the JNK signaling pathway and stemness-associated biomarkers was not performed. Moreover, SAL has been shown to inhibit CSCs via blocking Wnt/beta-catenin signaling (46). So the Wnt/beta-catenin signaling or other more CSC associated mechanisms and stemness-associated biomarkers should be performed in the future.

In conclusion, the combination of SAL and 17-AAG is likely to activate the JNK signaling pathway through the production of ROS, to induce apoptosis and inhibit autophagy, thus affecting the proliferation of breast cancer cells or CSCs (Fig. 7).

\section{Acknowledgements}

Not applicable.

\section{Funding}

The present study was supported in part by grants from the National Science and Technology Major Project (grant no. 2015ZX09501-009), National Natural Science Foundation (grant nos. 81760484, 31571469 and 81872349), Scientific Research Plan Projects of Shaanxi Education Department (grant no. 19JK0970), Scientific research project of Yan'an University (grant no. YDQ2019-38) and Free Exploring Projects of State Key Laboratory of Cancer Biology (grant no. CBSKL2014Z08)

\section{Availability of data and materials}

The datasets used and/or analyzed during the current study are available from the corresponding author on reasonable request.

\section{Authors' contributions}

BW conceived the idea for the study and performed the preliminary experiments. DH performed the experiment and prepared the original draft. JD analyzed and interpreted the data. LL and JZ contributed to the conception and design of the study and revised the final manuscript. All authors read and approved the final version of the manuscript submitted for publication. LL and JZ confirm the authenticity of all the raw data.

\section{Ethics approval and consent to participate}

Not applicable.

\section{Patient consent for publication}

Not applicable.

\section{Competing interests}

The authors declare that they have no competing interests.

\section{References}

1. Siegel RL, Miller KD, Fuchs HE and Jemal A: Cancer statistics, 2021. CA Cancer J Clin 71: 7-33, 2021.

2. Chaudhary LN, Wilkinson KH and Kong A: Triple-negative breast cancer: Who should receive neoadjuvant chemotherapy? Surg Oncol Clin N Am 27: 141-153, 2018.

3. Lee A and Djamgoz MBA: Triple negative breast cancer: Emerging therapeutic modalities and novel combination therapies. Cancer Treat Rev 62: 110-122, 2018.

4. Al-Mahmood S, Sapiezynski J, Garbuzenko OB and Minko T: Metastatic and triple-negative breast cancer: Challenges and treatment options. Drug Deliv Transl Res 8: 1483-1507, 2018.

5. Tang Y, Wang Y, Kiani MF and Wang B: Classification, treatment strategy, and associated drug resistance in breast cancer. Clin Breast Cancer 16: 335-343, 2016.

6. Turdo A, Veschi V, Gaggianesi M, Chinnici A, Bianca P, Todaro $M$ and Stassi G: Meeting the challenge of targeting cancer stem cells. Front Cell Dev Biol 7: 16, 2019.

7. Mummery C, Wilmut SI, van de Stolpe A and Roelen BAJ: Stem cells in cancer and cancer stem cells. In: Stem Cells. Vol 27. Academic Press, Cambridge, MA, pp237-256, 2011.

8. Dewangan J, Srivastava S and Rath SK: Salinomycin: A new paradigm in cancer therapy. Tumour Biol 39: 1010428317695035, 2017.

9. Gupta PB, Onder TT, Jiang G, Tao K, Kuperwasser C, Weinberg RA and Lander ES: Identification of selective inhibitors of cancer stem cells by high-throughput screening. Cell 138: 645-659, 2009.

10. Zhou J, Liu S, Wang Y, Dai W, Zou H, Wang S, Zhang J and Pan J: Salinomycin effectively eliminates cancer stem-like cells and obviates hepatic metastasis in uveal melanoma. Mol Cancer 18: 159, 2019.

11. Zhao Y, Zhao W, Lim YC and Liu T: Salinomycin-loaded gold nanoparticles for treating cancer stem cells by ferroptosis-induced cell death. Mol Pharm 16: 2532-2539, 2019.

12. Miyazaki Y, Shibuya M, Sugawara H, Kawaguchi O and Hirsoe C: Salinomycin, a new polyether antibiotic. J Antibiot (Tokyo) 27: 814-821, 1974.

13. Qin LS, Jia PF,Zhang ZQ and Zhang SM: ROS-p53-cyclophilin-D signaling mediates salinomycin-induced glioma cell necrosis. J Exp Clin Cancer Res 34: 57, 2015.

14. Kim KY, Lee SG, Baek SY, Lee EH, Jang EJ, Lee JH, Ahn SC, Chang JH, Oh TW, Kim SH, et al: Salinomycin ameliorates oxidative hepatic damage through AMP-activated protein kinase, facilitating autophagy. Toxicol Appl Pharmacol 360: 141-149, 2018.

15. Jiang J, Li H, Qaed E, Zhang J, Song Y, Wu R, Bu X, Wang Q and Tang Z: Salinomycin, as an autophagy modulator-a new avenue to anticancer: A review. J Exp Clin Cancer Res 37: 26, 2018.

16. Fuchs D, Daniel V, Sadeghi M, Opelz G and Naujokat C: Salinomycin overcomes ABC transporter-mediated multidrug and apoptosis resistance in human leukemia stem cell-like KG-1a cells. Biochem Biophys Res Commun 394: 1098-1104, 2010 . 
17. Wang Z, Zhou L, Xiong Y, Yu S, Li H, Fan J, Li F, Su Z, Song J, Sun Q, et al: Salinomycin exerts anti-colorectal cancer activity by targeting the $\beta$-catenin/T-cell factor complex. Br J Pharmacol 176: 3390-3406, 2019.

18. Kim KY, Park KI, Kim SH, Yu SN, Park SG, Kim YW, Seo YK, Ma JY and Ahn SC: Inhibition of autophagy promotes salinomycin-induced apoptosis via reactive oxygen species-mediated $\mathrm{PI} 3 \mathrm{~K} / \mathrm{AKT} / \mathrm{mTOR}$ and ERK/p38 MAPK-dependent signaling in human prostate cancer cells. Int J Mol Sci 18: 1088, 2017.

19. Talaei S, Mellatyar H, Asadi A, Akbarzadeh A, Sheervalilou R and Zarghami N: Spotlight on 17-AAG as an Hsp90 inhibitor for molecular targeted cancer treatment. Chem Biol Drug Des 93: 760-786, 2019

20. Hirakawa H, Fujisawa H, Masaoka A, Noguchi M, Hirayama R, Takahashi M, Fujimori A and Okayasu R: The combination of Hsp90 inhibitor 17AAG and heavy-ion irradiation provides effective tumor control in human lung cancer cells. Cancer Med 4: 426-436, 2015.

21. Moon HJ, Park SY, Lee SH, Kang CD and Kim SH: Nonsteroidal anti-inflammatory drugs sensitize CD44-overexpressing cancer cells to Hsp90 inhibitor through autophagy activation. Oncol Res 27: 835-847, 2019.

22. Xie Q, Wondergem R, Shen Y, Cavey G, Ke J, Thompson R, Bradley R, Daugherty-Holtrop J, Xu Y, Chen E, et al: Benzoquinone ansamycin 17AAG binds to mitochondrial voltage-dependent anion channel and inhibits cell invasion. Proc Natl Acad Sci USA 108: 4105-4110, 2011.

23. Walker T, Mitchell C, Park MA, Yacoub A, Rahmani M, Häussinger D, Reinehr R, Voelkel-Johnson C, Fisher PB, Grant S and Dent P: 17-allylamino-17-demethoxygeldanamycin and MEK1/2 inhibitors kill GI tumor cells via Ca2+-dependent suppression of GRP78/BiP and induction of ceramide and reactive oxygen species. Mol Cancer Ther 9: 1378-1395, 2010.

24. Piredda ML, Gaur G, Catalano G, Divona M, Banella C, Travaglini S, Puzzangara MC, Voso MT, Lo-Coco F and Noguera NI: PML/RARA inhibits expression of HSP90 and its target AKT. Br J Haematol 184: 937-948, 2019.

25. He D, Wo B and Zhao JM: Effect of salinomycin on the proliferation and apoptosis of triple negative breast cancer cell line MDA-MB-231 by target-regulating ALDH. Chinese J Oncol Prevention Treatment 12: 7, 2020.

26. Jafari-Gharabaghlou D, Pilehvar-Soltanahmadi Y, Dadashpour M, Mota A, Vafajouy-Jamshidi S, Faramarzi L, Rasouli S and Zarghami N: Combination of metformin and phenformin synergistically inhibits proliferation and hTERT expression in human breast cancer cells. Iran J Basic Med Sci 21: 1167-1173, 2018.

27. Chatran M, Pilehvar-Soltanahmadi Y, Dadashpour M Faramarzi L, Rasouli S, Jafari-Gharabaghlou D, Asbaghi N and Zarghami N: Synergistic anti-proliferative effects of metformin and silibinin combination on T47D breast cancer cells via hTERT and cyclin D1 inhibition. Drug Res (Stuttg) 68: 710-716, 2018.

28. Livak KJ and Schmittgen TD: Analysis of relative gene expression data using real-time quantitative PCR and the 2(-Delta Delta C(T)) method. Methods 25: 402-408, 2001

29. Kanehisa M, Furumichi M, Tanabe M, Sato Y and Morishima K: KEGG: New perspectives on genomes, pathways, diseases and drugs. Nucleic Acids Res 45: D353-D361, 2017.

30. Kanehisa M, Sato Y and Morishima K: BlastKOALA and GhostKOALA: KEGG tools for functional characterization of genome and metagenome sequences. J Mol Biol 428: 726-731, 2016

31. Verdoodt B, Vogt M, Schmitz I, Liffers ST, Tannapfel A and Mirmohammadsadegh A: Salinomycin induces autophagy in colon and breast cancer cells with concomitant generation of reactive oxygen species. PLoS One 7: e44132, 2012.
32. Park E and Chung SW: ROS-mediated autophagy increases intracellular iron levels and ferroptosis by ferritin and transferrin receptor regulation. Cell Death Dis 10: 822, 2019.

33. Zheng Q, Li Q, Zhao G, Zhang J, Yuan H, Gong D, Guo Y, Liu X, $\mathrm{Li} \mathrm{K}$ and Lin P: Alkannin induces cytotoxic autophagy and apoptosis by promoting ROS-mediated mitochondrial dysfunction and activation of JNK pathway. Biochem Pharmacol 180: 114167,2020

34. Su LJ, Zhang JH, Gomez H, Murugan R, Hong X, Xu D, Jiang F and Peng ZY: Reactive oxygen species-induced lipid peroxidation in apoptosis, autophagy, and ferroptosis. Oxid Med Cell Longev 2019: 5080843, 2019.

35. Wang S, Li Z, Liu W, Wei G, Yu N and Ji G: Neohesperidin induces cell cycle arrest, apoptosis, and autophagy via the ROS/JNK signaling pathway in human osteosarcoma cells. Am J Chin Med 49: 1251-1274, 2021.

36. Zhang J, Wang X, Vikash V, Ye Q, Wu D, Liu Y and Dong W: ROS and ROS-mediated cellular signaling. Oxid Med Cell Longev 2016: 4350965, 2016.

37. Zhang ZD, Yang YJ, Liu XW, Qin Z, Li SH and Li JY: Aspirin eugenol ester ameliorates paraquat-induced oxidative damage through ROS/p38-MAPK-mediated mitochondrial apoptosis pathway. Toxicology 453: 152721, 2021.

38. Diwanji N and Bergmann A: An unexpected friend-ROS in apoptosis-induced compensatory proliferation: Implications for regeneration and cancer. Semin Cell Dev Biol 80: 74-82, 2018.

39. Liu J, Chang F, Li F, Fu H, Wang J, Zhang S, Zhao J and Yin D: Palmitate promotes autophagy and apoptosis through ROS-dependent JNK and p38 MAPK. Biochem Biophys Res Commun 463: 262-267, 2015

40. Peña-Blanco A and García-Sáez AJ: Bax, Bak and beyondmitochondrial performance in apoptosis. FEBS J 285: 416-431, 2018.

41. Gu J, Zhan AJ, Jiang JL, Chen Y, Xu J, Ye L and Mao MG: Conserved function of pacific cod caspase-3 in apoptosis. Gene 732: 144370, 2020.

42. Tsapras P and Nezis IP: Caspase involvement in autophagy. Cell Death Differ 24: 1369-1379, 2017.

43. Liu X, Li Q, Sun L, Chen L, Li Y, Huang B, Liu Y and Jiang C: miR-30e-5p regulates autophagy and apoptosis by targeting Beclin1 involved in contrast-induced acute kidney injury. Curr Med Chem 28: 7974-7984, 2021.

44. Uriarte SM, Joshi-Barve S, Song Z, Sahoo R, Gobejishvili L, Jala VR, Haribabu B, McClain C and Barve S: Akt inhibition upregulates FasL, downregulates c-FLIPs and induces caspase-8-dependent cell death in Jurkat T lymphocytes. Cell Death Differ 12: 233-242, 2005

45. Oral O, Oz-Arslan D, Itah Z, Naghavi A, Deveci R, Karacali S and Gozuacik D: Cleavage of Atg3 protein by caspase- 8 regulates autophagy during receptor-activated cell death. Apoptosis 17: 810-820, 2012

46. Mao J, Fan S, Ma W, Fan P, Wang B, Zhang J, Wang H, Tang B, Zhang Q, Yu X, et al: Roles of Wnt/ $\beta$-catenin signaling in the gastric cancer stem cells proliferation and salinomycin treatment. Cell Death Dis 5: e1039, 2014.

This work is licensed under a Creative Commons Attribution-NonCommercial-NoDerivatives 4.0 International (CC BY-NC-ND 4.0) License. 